\title{
PERFIL CLÍNICO DE ADOLESCENTES QUE TÊM AIDS
}

\author{
Aline Cammarano Ribeiro무, Cristiane Cardoso de Paula², Eliane Tatsch Neves ${ }^{3}$, Stela Maris de Mello Padoin ${ }^{4}$
}

\begin{abstract}
RESUMO: Nesta pesquisa, objetivou-se caracterizar os adolescentes que têm a síndrome da imunodeficiência adquirida, assistidos em um hospital de ensino do Sul do Brasil, segundo sua história e evolução clínica. Pesquisa quantitativa, documental com abordagem descritiva, teve como critérios de inclusão idade entre 10-19 anos em tratamento antirretroviral, totalizando 39 prontuários. Com aprovação pelo Comitê de Ética em Pesquisa, deu-se a coleta de dados, utilizando formulário próprio; os dados foram submetidos ao tratamento estatístico pelo Programa Statistica Analisys System. Os resultados apontaram fragilidade clínica pelo comprometimento imunológico, vulnerabilidade às doenças oportunistas, necessidade de seguimento clínico e laboratorial permanentes, adesão ao tratamento, efeitos adversos e falhas terapêuticas. A partir desses resultados, salienta-se o compromisso de atender as demandas específicas da condição sorológica e da fase de crescimento e desenvolvimento própria da adolescência.
\end{abstract}

PALAVRAS-CHAVE: Saúde do adolescente; Síndrome da imunodeficiência adquirida; Enfermagem pediátrica.

\section{CLINICAL PROFILE OF TEENS THAT HAVE AIDS}

\begin{abstract}
This study aimed to characterize adolescents who have acquired the immunodeficiency syndrome, assisted in a teaching hospital in southern Brazil, according to their history and clinical course. It is a quantitative and documentary research, of a descriptive approach. It had as inclusion criteria the aged from 10-19 years, in antiretroviral treatment, totaling 39 patient records. Data collection was performed after the approval by the Ethics in Research Committee and using a proper form. Data were treated statistically by Statistica Analysis System. The results showed clinical fragility due to immunological impairment, vulnerability to opportunistic diseases, the need for permanent clinical and laboratory follow up, adherence to the treatment, adverse effects and treatment failure. From these results, the commitment to meet the specific demands of HIV status and stage of growth and development of their own adolescence is pointed out.
\end{abstract}

KEYWORDS: Adolescent health; Acquired immunodeficiency syndrome; Pediatric nursing.

\section{PERFIL CLÍNICO DE ADOLESCENTES CON SIDA}

RESUMEN: En esta investigación, se objetivó caracterizar los adolescentes que tienen el síndrome de la inmunodeficiencia adquirida, atendidos en un hospital de enseñanza del Sur del Brasil, según su historia y evolución clínica. Investigación cuantitativa, documental con abordaje descriptivo, tuvo como criterios de inclusión edad entre 10-19 años en tratamiento antirretroviral, totalizando 39 prontuarios. Con aprobación por el Comité de Ética en Investigación, se dió la colecta de datos, utilizando formulario propio, los datos fueron sometidos al tratamiento estadístico por el Programa Statistica Analisys System. Los resultados apuntaron fragilidad clínica por el comprometimiento inmunológico, vulnerabilidad a las enfermedades oportunistas, necesidad de seguimiento clínico y de laboratorio permanentes, adhesión al tratamiento, efectos adversos y fallas terapéuticas. A partir de esos resultados, se resalta el compromiso de atender las demandas específicas de la condición serológica y de la fase de crecimiento y desarrollo propia de la adolescencia.

PALABRAS CLAVE: Salud del adolescente; Síndrome de la inmunodeficiencia adquirida; Enfermería pediátrica.

${ }^{1}$ Enfermeira. Mestranda do Programa de Pós-Graduação em Enfermagem da Universidade Federal de Santa Maria-UFSM. Membro do Grupo de Pesquisa Cuidado à Saúde das Pessoas, Famílias e Sociedade.

${ }^{2}$ Enfermeira Pediatra. Doutora em Enfermagem. Professor Adjunto do Departamento de Enfermagem da UFSM. Líder do Grupo de Pesquisa Cuidado à Saúde das Pessoas, Famílias e Sociedade.

${ }^{3}$ Enfermeira Pediatra. Doutora em Enfermagem. Professor Adjunto no Departamento de Enfermagem da UFSM. Membro do Grupo de Pesquisa Cuidado à Saúde das Pessoas, Famílias e Sociedade.

${ }^{4}$ Enfermeira. Doutora em Enfermagem. Professor Adjunto no Departamento de Enfermagem da UFSM. Líder do Grupo de Pesquisa Cuidado à Saúde das Pessoas, Famílias e Sociedade.

Autor correspondente:

Aline Cammarano Ribeiro

Universidade Federal de Santa Maria

Rua Tuiuti, 2210 - 97050-420 - Santa Maria-RS, Brasil

Recebido: 15/11/09

E-mail: lilicammarano@yahoo.com.br

Aprovado: 11/04/10

Cogitare Enferm. 2010 Abr/Jun; 15(2):256-62 


\section{INTRODUÇÃO}

A infecção pelo vírus da imunodeficiência humana (HIV) e o adoecimento pela síndrome da imunodeficiência adquirida (aids) configuram-se como temáticas contemporâneas nas ciências da saúde e sociais, por sua natureza sociológica, política, econômica e clínico-epidemiológica. Elas apontam para necessidade de cuidados em saúde que contribuam para a melhoria das condições de vida das pessoas que têm esse tipo de enfermidade.

A evolução desse agravo à saúde no Brasil apontou a interface da problemática clínica e epidemiológica com a social e política, como reflexo das mudanças quantitativas e qualitativas que ocorreram no perfil da epidemia. Os casos notificados evidenciam uma tendência de juvenização e envelhecimento desta. Destaca-se que a juvenização é marcada pelos casos de aids notificados por idade, entre 1983-2008, na sua distribuição entre crianças e adolescentes: 9.929 casos ocorreram entre menores de cinco anos, 3.083 entre cinco e 12 anos, e 9.331 entre 13 e 19 anos. A transmissão vertical corresponde a $90 \%$ de casos entre os menores de cinco anos ${ }^{(1)}$.

Isso indica que a população de adolescentes que têm HIV/aids é composta por dois grupos, segundo a categoria de exposição do HIV: transmissão vertical e transmissão horizontal ${ }^{(2)}$. O primeiro grupo nasceu infectado pelo vírus devido à condição sorológica materna positiva ao HIV, o segundo grupo infectou-se por via sexual ou uso de drogas, caracterizando a transmissão horizontal.

O que os dois grupos têm em comum são as vivências decorrentes de uma doença, ainda estigmatizante e sem cura, que determina uma necessidade especial de saúde: a dependência de tecnologia medicamentosa do tratamento antirretroviral (TARV) $)^{(3-7)}$, associada à demanda de acompanhamento permanente de saúde.

Para o acompanhamento do processo de adoecimento é importante uma atenção específica às necessidades especiais de saúde dos adolescentes com aids, tendo em vista a fase de crescimento e modificações em seu metabolismo e composição corporal. Esse acompanhamento, de modo frequente e permanente, fornece informações aos profissionais que são relevantes para condutas de tratamento e para o alcance de resultados eficientes. Os adolescentes devem ser avaliados a cada dois ou três meses, dependendo de sua condição de vida, seja na dimensão biológica, psi- cológica, clínica, entre outras. Ou seja, a cada consulta, suas demandas de saúde devem ser reavaliadas, pois nessa fase suas condições podem mudar rapidamente ${ }^{(2)}$.

O seguimento ambulatorial é uma modalidade assistencial do acompanhamento de saúde para a redução das internações hospitalares que tem resultados significativos no tratamento. Inclui realização de exames laboratoriais e físico, retirada de medicamentos e avaliação da adesão $0^{(2,8)}$.

Esse seguimento pode acontecer por meio de consultas individuais ou atividades grupais, permeado de atenção multiprofissional. Os adolescentes que demonstrarem interesse têm a possibilidade de integrar-se nas atividades grupais, acompanhados de suas famílias ou pessoas que conhecem seu diagnóstico e participam do seu cuidado à saúde. Destaca-se que alguns serviços de saúde desenvolvem grupos de apoio ou terapêuticos específicos para os adolescentes. No entanto, em outros serviços, os adolescentes continuam sendo atendidos junto com as crianças ou com os adultos $^{(9)}$.

Para tanto, o objetivo desta pesquisa foi caracterizar os adolescentes que têm aids assistidos no ambulatório de um hospital de ensino da região sul do Brasil, segundo a sua história e evolução clínica. A finalidade é a de obter subsídios para desenvolver estratégias para o seu cuidado e para atender, não só as demandas de desenvolvimento próprios da fase da adolescência, como também as de adoecimento advindas da condição sorológica.

\section{METODOLOGIA}

Esta é uma pesquisa de abordagem quantitativa do tipo exploratório descritivo, utilizando-se do estudo retrospectivo para sustentar a busca de informação em documentos e registros de eventos já acontecidos, de forma sistematizada.

O projeto foi aprovado no Comitê de Ética em Pesquisa da instituição (processo: 23081.009010/200819).

Os dados foram coletados de julho a setembro de 2008, e o campo de pesquisa foi um hospital de ensino localizado em um município do interior do Estado do Rio Grande do Sul, que oferece serviço de referência para o atendimento de pacientes que têm HIV/aids a toda macrorregião centro-oeste do Estado. O Serviço de Infectologia desse hospital atende uma clientela de diferentes faixas etárias por meio de dois ambulatórios, um de pediatria e o outro para adultos. 
A partir dos critérios de inclusão - ter idade entre 10 e 19 anos e estar em tratamento antirretroviral - foram acessados 39 prontuários. Assim, foi composta a amostra de conveniência com a totalidade dos documentos disponíveis, ou seja, não houve perdas.

Para coletar os dados foi utilizado um roteiro estruturado com 30 questões sobre história clínica e evolução clínica. A primeira compôs-se pelas informações acerca das categorias: exposição, diagnóstico laboratorial, agendamento de consultas, assiduidade ao serviço de saúde e acompanhamento específico do crescimento e desenvolvimento. A segunda compôsse de informações sobre: esquema medicamentoso, tempo de uso do TARV, mudanças na terapia, efeitos adversos, uso de medicamentos profiláticos, infecções associadas/oportunistas e internações hospitalares.

Após o levantamento de dados, foi desenvolvida a tabulação e a análise dos dados com emprego da estatística descritiva. Foi utilizado como recurso o programa Statistica Analisys System (SAS versão 8,02 ) e os resultados foram apresentados segundo suas frequências simples.

\section{RESULTADOS E DISCUSSÃO}

Quanto à procedência dos 39 adolescentes, têm-se 48,72\% do município de Santa Maria, dentre os quais foi identificado o registro de três óbitos, que tiveram como causas: insuficência respiratória aguda, tumor no sistema nervoso central e uma não foi identificada no prontuário.

No que se refere às características demográficas, $56,41 \%$ são do sexo feminino; 61,54 \% estão na faixa etária de 10 a 12 anos; quanto à categoria de exposição, em $61,54 \%$ foi transmissão vertical do HIV; em 64,10\% o diagnóstico laboratorial ocorreu na infância, conforme tabela 1.

O índice de adolescentes infectados por transmissão vertical permite refletir sobre os avanço da tecnologia medicamentosa profilática na gestação e no parto e, também, a respeito da eficácia do TARV para diminuir a morbidade e mortalidade de crianças que nasceram infectadas pelo HIV.

OTARV modificou o curso da epidemia de aids, alterando sua evolução e tendências. As alterações no padrão dessa epidemia são resultado, também, das ações de prevenção e controle da infecção, da profilaxia e do manejo clínico das infecções oportunistas. Somadas às modalidades assistenciais para redução das internações hospitalares, como a assistência ambu- latorial especializada. Em conjunto, tiveram impacto sobre a morbidade e mortalidade de pessoas infectadas pelo HIV ou que têm aids ${ }^{(8)}$. Portanto, a aids é considerada um doença crônica ${ }^{(7)}$, evidenciando novas perspectivas como a transição da infância para adolescência das crianças que têm aids por transmissão vertical $^{(10-12)}$.

Tabela 1 - Características dos adolescentes que têm aids cadastrados em ambulatório de hospital de ensino no Sul do Brasil, 1996-2008.

\begin{tabular}{lcc}
\hline Variáveis & $\begin{array}{c}\text { Frequência } \\
\text { absoluta }\end{array}$ & $\begin{array}{c}\text { Frequência } \\
\text { relativa }\end{array}$ \\
\hline Sexo & 22 & 56,41 \\
Feminino & 17 & 43,59 \\
Masculino & & \\
Faixa etária & 24 & 61,54 \\
$10-12$ & 10 & 25,64 \\
$13-15$ & 4 & 10,25 \\
$16-18$ & 1 & 2,56 \\
19 anos & & \\
Categoria de exposição & 24 & 61,54 \\
Transmissão vertical & 3 & 7,70 \\
Transmissão sexual & 1 & 2,56 \\
Transfusão sanguínea & 11 & 28,20 \\
Não identificado & & \\
Idade do diagnóstico & & 64,10 \\
Infầncia & 25 & 15,38 \\
(3 meses a 9 anos) & & 20,52 \\
Adolescência & 6 & \\
(10 a 19 anos) & 8 & \\
Não identificado & & \\
\hline
\end{tabular}

O grupo de aquisição vertical contrariou a ciência, na medida em que não se antevia perspectiva de vida para as crianças infectadas desta forma "fabricamos uma criança vítima, criança drama e um adolescente que não existiria”(10:29). Entretanto, venceram a infância e adentraram na adolescência com suas vulnerabilidades ${ }^{(11)}$, decorrentes das implicações biológicas e sociais específicas dessa fase do desenvolvimento humano, somadas às necessidades especiais de saúde da condição sorológica ${ }^{(12)}$.

Na categoria de exposição da infecção por transmissão horizontal os resultados evidenciaram que os adolescentes continuam infectando-se por exposição 
sexual e sanguínea. A via sexual é decorrente, principalmente, de relação desprotegida, e a exposição sanguínea resultante do uso de drogas injetáveis ${ }^{(1)}$.

Quanto ao diagnóstico laboratorial, o grupo de adolescentes de aquisição vertical do HIV teve diagnóstico na infância ou adolescência. Na infância, o diagnóstico precoce acontece quando a mãe mantém seguimento de puericultura, possibilitando o acompanhamento clínico e laboratorial. Outra possibilidade se deve ao aparecimento de sintomas, que segue o padrão bimodal: precoce (quando a criança tem, em média, quatro meses de idade) e tardia (quando a criança tem, em média, seis anos de idade) ${ }^{(2,13)}$, situação que converge com os resultados desta pesquisa.

A necessidade especial de saúde decorrente da Aids demanda um seguimento ambulatorial permanente para acompanhamento imunológico, clínico e farmacológico. Para tanto, o serviço de saúde precisa de profissionais capacitados para atender os adolescentes e suas famílias, objetivando a redução da morbidade e mortalidade.

O grupo de aquisição horizontal pode ter diagnóstico laboratorial: por investigação sorológica com testagem anti-HIV voluntária, por investigação clínica de doenças recorrentes, ou por situações de exposição como nos casos de violência e gestação na adolescência $^{(2)}$. A investigação clínica ainda está relacionada a internações recorrentes sem causa determinada, o que leva a equipe de saúde a investigar a sorologia, com consentimento da família quando se trata de crianças e adolescentes ${ }^{(2,13)}$.

Quanto ao agendamento de consultas específicas 46,15\% tiveram entre 4-6 consultas na infectologia no último ano. O seguimento ambulatorial em serviço de referência mostra a necessidade de acompanhamento de saúde contínuo e permanente dos adolescentes que têm aids. O manejo da doença é complexo e exige que sejam considerados fatores específicos como marcadores biológicos, evolução da doença, adesão ao TARV, entre outros ${ }^{(2)}$.

As consultas podem acontecer com a presença de familiares/cuidadores, porém, é importante um momento individualizado, um espaço apenas do adolescente, a fim de que possa sentir-se à vontade para conversar sobre dúvidas da sua fase de desenvolvimento, da condição sorológica e no que tange ao tratamento e à prevenção da transmissão do HIV.

A relação entre profissional, adolescente e família aponta a necessidade de vínculos no serviço de saúde, para haver segurança e compromisso no percurso clínico, social e existencial. No entanto, os serviços de referência são, na maioria, hospitais universitários, para os quais ocorre grande fluxo de estudantes e profissionais, o que fragiliza os vínculos necessários para continuidade de um bom tratamento.

O grupo de aquisição vertical do HIV se caracteriza por apresentar vínculos sólidos com o serviço de saúde e cuidadores, pois geralmente estão em tratamento há muitos anos. Os principais problemas encontrados na assistência a esse grupo são revelação do diagnóstico, orfandade, desestruturação familiar e início da atividade sexual. O grupo de aquisição horizontal apresenta vínculos frágeis com o serviço de saúde e cuidadores e, frequentemente, apresenta agravos sociais diversos, dificuldade em buscar os serviços de saúde, problemas escolares e de inserção profissional ${ }^{(2)}$.

Quanto à assiduidade no serviço de saúde, 66,67\% foram assíduos a todas as consultas. Esses resultados mostram que o acesso ao serviço de referência está sendo efetivo e que há compreensão da necessidade do seguimento para a manutenção da saúde. No entanto, alguns adolescentes ainda têm deficit na continuidade do acompanhamento de sua saúde, sendo que as prováveis causas não estão especificadas nos prontuários. Esse deficit poderia ser compreendido pela influência da estrutura do serviço quanto a distância, espaço próprio para essa população, vínculo com a equipe, preparo dessa para atendar as demandas específicas do desenvolvimento, interferência no cotidiano escolar e social, entre outros fatores.

Cabe aos profissionais aprimorar o acesso dos adolescentes ao serviço de saúde para favorecer, principalmente, o acompanhamento clínico e a adesão ao TARV, que são ações decisivas à manutenção da saúde dos adolescentes. Cabe ainda, promover a educação em saúde para prevenir a transmissão do HIV $^{(2)}$. Nesse sentido, percebe-se a necessidade de um espaço próprio ao adolescente que têm aids, com estratégias de manutenção e recuperação da saúde por ações educativas e culturais que envolvam o indivíduo na promoção de sua autonomia, para o cuidado de si.

A abordagem com o adolescente, no serviço de saúde, deve ser realizada de maneira singular e humanizada, com uma equipe multiprofissional sensibilizada que possa identificar as mudanças físicas, cognitivas e sociais específicas da idade, para melhor enfretamento dos desafios da doença. Assim, o serviço de saúde onde o adolescente realiza acompanhamento clínico deve dispor de profissionais capacitados, rotinas 
de atendimento, aconselhamento, grupos educativos, espaço físico adequado, referência e contrarreferência eficiente. Essas ações são necessárias para não haver fragmentação na assistência ao adolescente e garantir qualidade em seu tratamento( ${ }^{(2)}$.

Quanto ao acompanhamento específico do crescimento e desenvolvimento, 79,49\% não tiveram acompanhamento específico, sendo que os prováveis motivos não estão especificados nos prontuários. Destacase a importância na atenção à puberdade e à sexualidade do adolescente, a fim de acompanhar o processo de crescimento e de mudanças próprias dessa fase, considerando sua variabilidade quanto ao início, duração e progressão até a maturidade sexual, atentando para os comprometimentos decorrentes da condição sorológica. Os resultados desta pesquisa evidenciam a preocupação com as questões clínicas da aids e demonstram certa negligencia quanto ao acompanhamento puberal.

Além disso, enfatiza-se que o papel do serviço de saúde não é o de controlar o exercício da sexualidade dos adolescentes que têm HIV/aids; mas sim fornecer subsídios para sua vida plena e segura, e promover a autonomia para o cuidado de $\mathrm{si}^{(2,14)}$.

Quanto à evolução clínica, o esquema de TARV foi singular a cada adolescente, conforme seus dados clínicos. Os dois grupos de adolescentes são acometidos com características distintas. No grupo de aquisição vertical, os adolescente, em sua maioria, são mais novos e, usualmente, encontram-se nos estágios iniciais da puberdade, podendo ocorrer retardo puberal e altera ções de desenvolvimento neurocognitivo. No grupo de aquisição horizontal, os adolescentes normalmente são mais velhos, estão usualmente nos estágios finais da puberdade e foram recentemente infectados ${ }^{(2)}$. Portanto, justifica-se a necessidade de acompanhar o crescimento e desenvolvimento por duas demandas: a própria da fase da adolescência, e que envolve transformações biológicas e sociais; e as demandas relacionadas à condição sorológica, que envolve o processo de adoecimento nessa fase. Em ambas, a prevenção da transmissão do HIV por relação sexual deve ser enfatizada.

Quanto ao esquema medicamentoso, 30,56\% têm tempo de TARV de um a três anos; $69,45 \%$ de 4 a 9 anos; e 90,32\% já tiveram mudanças no TARV devido à indicação terapêutica ou resistência medicamentosa. Considerando os intervalos de idade de 10-14 anos e 15-19 anos, em ambos a média de tempo de tratamento foi de 2,5 anos. As mundanças na terapia são indicadas em casos de intolerância, toxicidade e falha terapêutica. Para o sucesso terapêutico, não se devem observar somente os níveis de carga viral; percebe-se a necessidade de um acompanhamento mais próximo ao adolescente para a identificação da adaptação do TARV. Quando necessário, deve ser realizada a genotipagem para melhor adequação do tratamento. Para modificação dos esquemas de ARV, vários fatores devem ser considerados como: crescimento e desenvolvimento; adaptação aos medicamentos; dados clínicos; contexto familiar e social ${ }^{(2,13)}$.

A terapia combinada de TARV mostrou-se singular a cada adolescente, pois este é um paciente em fase de crescimento e modificação do metabolismo, e composição corporal com ritmo acelerado. Por isso, necessita de ajuste frequente do tratamento, segundo estadiamento da puberdade de Tanner e/ou resistência medicamentosa ${ }^{(13)}$. A indicação de esquema medicamentoso para o TARV dos adolescentes varia conforme o momento em que aconteceu a infecção e a evolução da doença. Parte dos adolescentes do grupo de aquisição vertical, geralmente em tratamento há muitos anos, atinge a adolescência exposta a múltiplos regimes de TARV. E apresenta vários efeitos adversos, com reduzidas opções terapêuticas, necessitando acesso a novas drogas, por vezes ainda não aprovadas para sua faixa etária. Em alguns casos, cuidados paliativos para a manutenção da qualidade de vida são prioritários. Os adolescentes do grupo de aquisição horizontal, comumente, estão em início de tratamento ${ }^{(2)}$.

Para a escolha da terapia combinada de TARV, utiliza-se o estadiamento de Tanner. Normatiza-se que adolescentes em estágio I e II, por estarem no início da puberdade, devem ser tratados com doses pediátricas; em estágio III e IV, por estarem no momento acentuado das transformações da puberdade, devem receber medicamento individualizado; e em estágio $\mathrm{V}$, em que a puberdade encontra-se consolidada, devem receber doses de adultos ${ }^{(2,13)}$. Assim, as transformações da adolescência devem estar associadas às características clínicas, para haver complementaridade e resolutividade nas ações de saúde desenvolvidas com o adolescente que tem aids.

A adesão constitui importante ação para a resolutividade do tratamento, configurando uma aliança terapêutica entre o profissional e o adolescente, podendo incluir a família. Por meio do uso sistemático dos medicamentos específicos, objetiva melhora clínica, aumento do tempo de vida e redução de falhas terapêuticas ${ }^{(3,4)}$. Além disso, possibilita benefícios como 
a regressão de manisfestações clínicas, doenças oportunistas, internações hospitalares e mortalidade, além de melhoria no processo de crescimento e desenvolvimento, na condição física e emocional e na vida dos adolescentes. Entretanto, há inúmeras dificuldades para alcançar a adesão.

Os efeitos adversos se configuram em fator de grande influência na adesão ao TARV ${ }^{(2,13)}$. No presente estudo, apresentaram efeitos adversos ao TARV, principalmente diarreia e vômito, 81,82\% dos adolescentes. É importante a compreensão, pelos adolescentes, do significado do tratamento proposto e seus possíveis efeitos, pois isto - juntamente com seu familiar/cuidador - contribui para o cuidado à saúde, no intuito de intensificar hábitos que mimizem os efeitos adversos $^{(2)}$. A compreensão pelo adolescente da importância do TARV conduz à minimização de impacto e de implicações negativas dos efeitos adversos, pois resultam em reclamações físicas e desconfortos que o jovem não sentia antes de iniciar o tratamento medicamentoso.

Os fatores associados à adesão referem-se, além das questões específicas do paciente, à doença, aos problemas sociais e ao serviço de saúde. Quanto ao paciente, depende de fatores sociodemográficos, inclusive da unidade familiar, como orfandade e institucionalização, além do conhecimento sobre a doença e, o tratamento, e a autonomia para o autocuidado. Quanto à doença, tem-se as variáveis relacionadas à presença ou ausência de sintomas, controle periódico, resultados laboratoriais, complexidade do esquema medicamentoso, tempo de tratamento, apresentação dos medicamentos, efeitos adversos, dentre outros ${ }^{(3-6)}$. O suporte social, as condições de habitação, renda, ocupação, emprego, discriminação, relação com a família, relações com os pares, isolamento social, rede social, dentre outros, deverão ser considerados. Quanto ao serviço de saúde, a adesão tem correlação com o acesso à capacitação profissional, à qualidade do cuidado, ao vínculo com profissionais, à retirada de medicamentos, entre outros. Somam-se às complicações, a baixa potência da(s) droga(s) da terapia combinada, toxicidade medicamentosa, resistência viral e necessidade de alta adesão ao tratamento ${ }^{(3-6)}$

Considera-se um caso não-aderente quando há interrupção do tratamento ou falha no cumprimento da prescrição, ou ainda quando o adolescente ingere o medicamento em menor ou maior quantidade do que a posologia indicada, omite doses ou altera intervalos de tempo determinados e não segue as recomendações dietéticas ${ }^{(4)}$.

Medicamento profilático associado ao TARV é utilizado em 51,28\%, dos adolescentes sendo em 36,36\% usado Bactrim ${ }^{\circledR}$. Já desenvolveram infecções associadas/oportunistas $84,21 \%$ dos casos, sendo pneumonia a mais frequente; 76,92\% necessitaram de internação hospitalar. O uso de medicamentos profiláticos também se mostra essencial à manutenção da saúde, devido ao comprometimento imunológico e risco elevado de infecções bacterianas, fúngicas, parasitárias e virais. A profilaxia visa, principalmente, a prevenção de pneumonia, frequente em pessoas soropositivas e que pode se manifestar com rapidez e alta letalidade. Quando o tratamento não é efetivo, poderá haver a necessidade de internações hospitalares, as quais poderão repercutir no comprometimento da saúde emocional $^{(13,15)}$.

No Brasil, evidencia-se que, pela universalidade do acesso ao TARV, o grupo de adolescentes de aquisição vertical está transitando da infância para adolescência e, consequentemente, para a idade adulta. A perspectiva de vida longa para as pessoas infectadas pelo HIV depende de muitos fatores, além da distribuição de medicamentos. Somam-se as intervenções singulares, sociais, políticas e legais em questões que envolvem o cotidiano pessoal, familiar, comunitário, profissional e de saúde.

Por fim, a caracterização da história e evolução clínica dos adolescentes que têm aids apontou que a condição de cronicidade da doença, mediada pela tecnologia medicamentosa do TARV e os avanços do atendimento à saúde, possibilitaram uma perspectiva de vida. Evidenciou-se a fragilidade clínica decorrente do processo de adoecimento, o qual influencia tanto na saúde quanto no crescimento e desenvolvimento para os dois grupos: de aquisição vertical ou horizontal do vírus.

\section{CONCLUSÕES}

A fragilidade clínica dos adolescentes que têm aids foi evidenciada por: exposição à infecção pelo HIV; comprometimento imunológico; manifestações sintomatológicas da doença; morbimortalidade por AIDS ou infecções associadas/oportunistas; internações hospitalares frequentes; necessidade de acompanhamento de saúde permanente; foram também percebidas as suas repercussões no processo de crescimento e de desenvolvimento puberal, que também está associada ao cotidiano medicamentoso, à adesão 
ao tratamento, à efetividade, aos efeitos adversos e limites terapêuticos.

Portanto, tem-se um duplo compromisso, atender as demandas específicas da condição sorológica e as demandas da fase de crescimento e desenvolvimento. É preciso implantar ações de promoção e manutenção da saúde e prevenção do adoecimento dessa população específica, e para tanto é imprescindível aprimorar o acesso ao serviço, o vínculo com os profissionais, a capacitação permanente da equipe multiprofissional, a construção de um espaço próprio para os adolescentes, adotar estratégias de educação em saúde e de cuidado coerentes com a fase da adolescência, subsídios para adesão ao TARV, entre outros. Objetiva-se um cuidado compartilhado entre a equipe da saúde, os familiares e os próprios adolescentes, com vistas ao desenvolvimento da autonomia para o cuidado de si.

A partir deste estudo, compreende-se que a Enfermagem pode contribuir como parte da equipe de saúde, nas ações de cuidado e educação em saúde. Destacam-se as consultas individuais ou em grupos, com a possibilidade de desenvolver uma assistência integral e humanizada, de modo que adolescentes recebam apoio para o cuidado à saúde que contemple as dimensões biológica, clínica, social, ética e subjetiva, para viver melhor e com qualidade.

Apesar das limitações encontradas na coleta de dados nos prontuários, que apresentam deficit de alguns registros, a caracterização dos adolescentes que têm aids apontou um perfil clínico com indicativos da história da infecção e evolução da doença, os quais evidenciaram sua fragilidade clínica.

\section{REFERÊNCIAS}

1. Ministério da Saúde(BR). Boletim Epidemiológico AIDS DST. Brasília; 2008. Ano V, n.1, p. 13,31. Brasília, 2008. Disponível: http://www.aids.gov.br

2. Ministério da Saúde (BR). Manual de rotinas para assistência de adolescentes vivendo com HIV/Aids. Brasília; 2006.

3. Reiners AAO, Azevedo RCS, Vieira MA, Arruda ALG. Produção bibliográfica sobre adesão/não-adesão de pessoas ao tratamento de saúde. Ciênc Saúde Coletiva 2008;13(Supl 2):2299-306.

4. Barroso LLMMB, Pereira KKCP, Almeida PPCA, Galvão MMTGG. Adesão ao tratamento com anti-retrovirais entre pacientes com aids. Online Braz J Nurs. [Internet]
2006;5(2). [acesso em 15 out 2009]. Disponível: http:// www. uff.br/objnursing/index.php/nursing

5. Colombrini MRC, Lopes MHBM, Figueiredo RM. Adesão à terapia antiretroviral para HIV/AIDS. Rev Esc Enferm USP 2006;40(4):576-81.

6. Costa LS, Latorre MRDO, Silva MH, Bertolini DV, Machado DM, Pimentel SR et al. Validação e reprodutibilidade de uma escala de auto-eficácia para adesão ao tratamento anti-retroviral em pais ou cuidadores de crianças e adolescentes vivendo com HIV/AIDS. J Pediatr. 2008;84(1):41-6.

7. Schaurich D, Coelho DF, Motta MGC. A cronicidade no processo saúde-doença: repensando a epidemia da AIDS após os anti-retrovirais. Rev Enferm UERJ 2006;14(3):455-62.

8. Brito AM, Souza JL, Luna CF, Dourado I. Tendência da transmissão vertical de aids após a terapia anti-retroviral no Brasil. Rev Saúde Pública 2006;40(Supl):9-17.

9. Paula CC, Cabral IE, Souza IEO. Cotidiano de crianças infectadas pelo HIV no adolescer: compromissos e possibilidades do cuidar de si. DST J. Bras Doenças Sex Transm. 2008;20(3-4):173-8.

10. Cruz EF. Infâncias, adolescências e aids. Educ Rev. 2007;23(46): 363-84.

11. Schaurich D, Medeiros HM, Motta MGC. Vulnerabilidades no viver de crianças com aids. R Enferm UERJ 2007;15(2):284-90.

12. Paula CC, Cabral IE, Souza IEO. O cotidiano do seradolescendo que tem aids: momento ou movimento existencial? Esc Anna Nery 2009;13(3):632-9.

13. Ministério da Saúde (BR). Guia de tratamento clínico da infecção pelo HIV em crianças. Brasília; 2007.

14. Camargo B, Botelho LJ. Aids, sexualidade e atitudes de adolescentes sobre proteção contra o HIV. Rev Saúde Pública 2007;41(1):61-8.

15. Maas T, Zagonel IPS. Transição de saúde-doença do ser adolescente hospitalizado. Cogitare Enferm. 2005;10(2):68-75. 